\title{
O uso de preservativos: a realidade de adolescentes e adultos jovens de um
}

\section{assentamento urbano}

\author{
Brenda Kelly Gonçalves Nunes ${ }^{1}$, Alessandra Dias Lemes Guerra ${ }^{2}$, Stéfany Martins Silva ${ }^{3}$, \\ Rafael Alves Guimarães ${ }^{4}$, Márcia Maria de Souza ${ }^{5}$, Sheila Araújo Teles ${ }^{6}$, Marcos André de Matos ${ }^{7}$
}

\footnotetext{
${ }^{1}$ Discente do curso de graduação em Enfermagem da Faculdade de Enfermagem da Universidade Federa de Goiás. Goiânia, GO, Brasil. E-mail:

brendakellynunes@gmail.com.

${ }^{2}$ Discente do curso de graduação em Enfermagem da Faculdade de Enfermagem da Universidade Federa de Goiás. Goiânia, GO, Brasil. E-mail: alediasguerra@gmail.com.

${ }^{3}$ Enfermeira. Goiânia, GO, Brasil. E-mail: fefy martins@hotmail.com.

${ }^{4}$ Enfermeiro, Mestre em Enfermagem. Goiânia, GO, Brasil. E-mail: rafaelalvesg5@gmail.com.

${ }^{5}$ Enfermeira, Doutora em Ciências da Saúde. Professor Adjunto da Universidade Federal de Goiás. Goiânia, GO, Brasil. Email: marcia@fen.ufg.br.

${ }^{6}$ Enfermeira, Doutora em Biologia Parasitária. Professor Associado da Universidade Federal de Goiás. Goiânia, GO, Brasil. E-mail: sheila.fen@gmail.com.

${ }^{7}$ Enfermeiro, Doutor em Ciências da Saúde. Professor Adjunto da Universidade Federal de Goiás. Goiânia, GO, Brasil. Email: marcosmatos@ufg.br.
}

\section{RESUMO}

Estudo com objetivo de estimar a prevalência e fatores associados ao uso de preservativo masculino em adolescentes e jovens de áreas de assentamento urbano de uma capital do centro-oeste brasileiro. Investigação de corte transversal, realizada em 105 adolescentes e jovens escolares de 12 a 24 anos, utilizando-se questionário estruturado. Do total de entrevistados, 61 (58,1\%) haviam iniciado a vida sexual e destes, apenas $38,3 \%$ relataram uso regular do preservativo. Escolaridade acima de seis anos $(p=0,02)$, acesso a informação sobre sexualidade com os pais $(p=0,05)$ e na escola $(p=0,04)$ foram fatores associados ao uso do preservativo. Os resultados deste estudo ratificam a importância de investimento em políticas de saúde que invistam no trabalho conjunto entre profissionais de saúde, família e instituições de ensino, visando minimizar a vulnerabilidade às Infecções Sexualmente Transmissíveis. Descritores: Medicina do Adolescente; Saúde Escolar; Enfermagem em Saúde Comunitária.

\section{INTRODUÇÃO}

O adolescer é marcado por intensas mudanças físicas, psíquicas e pessoais, no qual os indivíduos consolidam valores e conceitos a partir de influências culturais, familiares, espirituais, pela mídia e, principalmente, por questões sociais ${ }^{(1)}$. É uma fase do desenvolvimento humano no qual há busca constante pela autonomia, por meio da autodeterminação pessoal, construindo sua identidade social e de 
gênero $^{(1-2)}$.

Também é caracterizada pelo início da vida sexual devido ao desenvolvimento corporal, acompanhado pelas descobertas das questões relacionadas à sexualidade humana, podendo ser um período de vulnerabilidade para exposição às doenças, pois os adolescentes e adultos jovens, em geral, se consideram em saúde plena e juventude intacta, adotando, assim, comportamentos sexuais inseguros ${ }^{(1-3)}$.

Embora ainda exista ampla disparidade em relação às concepções acerca do recorte etário de adolescência e juventude, tendo em vista se tratar de uma construção sócio-histórica, econômica, cultural e relacional, esse período de transição entre a infância e a vida adulta é marcado por comportamentos e atitudes que potencializam suas vulnerabilidades. De tal modo, independentemente da faixa etária, este grupo populacional têm vivido um processo de invisibilidade social que os coloca à margem das políticas públicas de saúde ${ }^{(3)}$.

A vulnerabilidade dos indivíduos está relacionada a um plano interdependente de determinação de caráter individual, social e programático, os quais integram valores, crenças, desejos, conhecimentos e comportamentos (âmbito individual); contextos de vida e valores morais (âmbito social); educação, cultura e saúde (âmbito programático), que irão interferir na exposição e/ou prevenção de doenças, tais como as Infecções Sexualmente Transmissíveis (IST), tornando-se, então, alvo de campanhas de prevenção e controle de IST pelos diferentes órgãos governamentais ${ }^{(4)}$. Em termos de prevenção o preservativo masculino é reconhecidamente o método de barreira mais eficaz das IST, bem como de uma gravidez não planejada, entretanto, sabe-se que o seu uso entre a população jovem ainda apresenta valores insatisfatórios ${ }^{(5-6)}$.

Portanto, é importante estudos acerca do uso do preservativo, em particular entre adolescentes e jovens de áreas de assentamento, uma vez que este é um grupo emergente com vulnerabilidade aos agravos em saúde, em especial aqueles relacionados à sexualidade humana, e ainda são escassos os estudos sobre a temática, sendo os poucos existentes voltados para indivíduos de assentamento urbano informal ${ }^{(7-8)}$. Além disso, o conhecimento da realidade que norteia esses indivíduos favorece o desenvolvimento de ações e política em saúde específica capazes de atuarem em suas necessidades, proporcionando prevenção contra as IST e gravidez não planejada, com visibilidade e equidade em relação à saúde da população jovem, em seus diversos contextos sociais.

Partindo desses argumentos, o objetivo do estudo foi estimar a prevalência e fatores associados ao uso regular do preservativo masculino entre adolescentes e jovens escolares de áreas de assentamento.

\section{MATERIAL E MÉTODOS}

Estudo analítico, de corte transversal, realizado com 105 adolescentes e jovens escolares de 12 a 24 anos, dos 192 matriculados em uma única Instituição de Ensino e residentes em assentamento urbano formal localizado na periferia de uma capital do centro-oeste brasileiro.

O referido assentamento foi idealizado para atender famílias que residiam em assentamentos informais, sendo desprovidos de pavimentação, rede de esgoto, segurança pública e áreas de lazer. 0 
assentamento conta com apenas uma Instituição de Ensino.

Como critério de inclusão participaram adolescentes ou jovens de faixa etária entre 12 a 24 anos, visando atender as concepções estabelecidas sobre o recorte etário de adolescência e juventude ${ }^{(3)}$, residentes no assentamento em estudo e que estivessem matriculados e frequentando a instituição de ensino do assentamento. Assim, participaram $54,7 \%$ do total de escolares assentados da Instituição de Ensino elegíveis para o estudo.

Os dados foram coletados no período de agosto a novembro de 2013 por meio de questionários contendo perguntas abertas e fechadas. Todos os adolescentes e jovens foram convidados a participar do estudo em sua instituição de ensino e remarcado um novo encontro juntamente com os pais e/ou responsáveis pelos menores. A coleta de dados ocorreu na Unidade Básica de Saúde (UBS) da região e na escola, a qual os adolescentes eram matriculados. A entrevista foi realizada em uma área reservada em ambos os locais, pelos pesquisadores e auxiliares de pesquisa previamente capacitados, os quais antes orientavam os participantes quanto à seriedade e o anonimato das respostas.

O roteiro de entrevista abordou questões sobre as características sociodemográficas: sexo, idade, escolaridade, estado civil, tempo de assentamento, renda familiar, religião e fatores para a adesão ao preservativo: sexarca, uso de tatuagem, uso de piercing, uso de drogas ilícitas e bebida alcoólica, acesso a informações sobre sexualidade com os pais, na escola e nos serviços de saúde e tipo de atendimento na UBS.

A variável de desfecho foi o uso regular do preservativo masculino nas relações sexuais, sendo que foi considerado como regular o uso do preservativo em toda e qualquer relação sexual, seja com parceria fixa ou não.

Os dados foram analisados por meio de um software. Para as variáveis contínuas foram calculados médias e desvio-padrão. Para verificar as associações entre o desfecho e as demais variáveis, realizou-se análise univariada, obtendo as razões de chance (Odds ratio) e os respectivos intervalos de confiança (IC 95\%). Os testes de $\chi^{2}$ e exato de Fisher foram utilizados para testar as diferenças entre as proporções. Valores de $p<0,05$ foram considerados estatisticamente significativos.

O projeto foi avaliado e aprovado pelo Comitê de Ética em Pesquisa da Universidade Federal de Goiás (no 365/11). Antes de iniciar a coleta havia a assinatura do Termo de Consentimento Livre e Esclarecido (TCLE) pelos responsáveis (menores de 18 anos) ou pelo próprio participante (maiores de 18 anos).

\section{RESULTADOS}

\section{Perfil sociodemográfico}

Participaram do estudo 105 adolescentes e jovens escolares de um assentamento urbano. Quanto ao sexo, 58,1\% eram do sexo masculino e 41,9\% feminino. A média de idade foi de 16,2 (DP \pm 3,32; Min.12; Máx. 24), com predomínio de adolescentes (12-18 anos; $73,3 \%)$.

A média de anos de escolaridade foi de 7,76 (DP£ 1,75; Min.: 3; Máx.: 12) e de tempo de assentamento 
foi de 2,73 (DP£0,94; Min.:1, Máx.: 5). Quanto à renda familiar 81,0\% dos participantes tinham renda de até três salários mínimos.

Vinte $(19,0 \%)$ indivíduos relataram não praticar nenhuma religião. Dos praticantes, $56,2 \%$ se declararam evangélicos.

\section{Uso do preservativo}

Do total de participantes, $60(40,6 \%)$ já tinham iniciado a vida sexual. Quanto ao uso do preservativo masculino nas relações sexuais, $38,3 \%$ relataram uso em todas as relações sexuais (uso regular) e $61,7 \%$ o uso inconsistente ou não adesão ao método de barreira.

A Tabela 1 apresenta os fatores associados ao uso do preservativo em análise univariada. Assim, o uso regular do preservativo nas relações sexuais foi associado à escolaridade superior a seis anos (OR: 9,3; IC 95\%: 1,1-77,9; $p=0,02$ ), acesso à informações sobre sexualidade na escola (OR: 1,9; IC 95\%: 1,1-3,6; $p=0,04$ ) e com os pais (OR: 1,9; IC 95\%: 1,0-3,5; p=0,05). Ainda, não possuir tatuagem (OR: 3,9; IC 95\%: 0,8-19,7; $p=0,08)$ permaneceu marginalmente associado ao uso de preservativo.

Tabela 1: Fatores associados ao uso regular do preservativo masculino em 60 adolescentes e jovens escolares de um assentamento urbano de Goiânia, GO, Brasil, 2013.

\begin{tabular}{|c|c|c|c|c|}
\hline \multirow{2}{*}{ Variável } & \multicolumn{2}{|c|}{ Uso regular do preservativo } & \multirow{2}{*}{ Oddsratio (IC 95\%) ${ }^{\dagger}$} & \multirow{2}{*}{$\mathbf{p}^{\S}$} \\
\hline & Sim/Total & (\%) & & \\
\hline \multicolumn{5}{|l|}{ Sexo } \\
\hline Feminino & $8 / 23$ & 34,8 & 1.0 & \\
\hline Masculino & $15 / 37$ & 40,5 & $1,3(0,4-3,8)$ & 0,70 \\
\hline \multicolumn{5}{|l|}{ Idade (anos) } \\
\hline $12-18$ & $14 / 34$ & 41,2 & 1.0 & \\
\hline $19-24$ & $9 / 26$ & 34,6 & $0,8(0,3-2,2)$ & 0,60 \\
\hline \multicolumn{5}{|l|}{ Estado civil } \\
\hline Casado & $2 / 7$ & 28,6 & 1.0 & \\
\hline Solteiro/separado & $21 / 53$ & 39,6 & $1,6(0,3-9,3)$ & 0,50 \\
\hline \multicolumn{5}{|l|}{ Escolaridade (anos) } \\
\hline$\leq 6$ & $1 / 12$ & 8,3 & 1.0 & \\
\hline$>6$ & $22 / 48$ & 45,8 & $9,3(1,1-77,9)$ & 0,02 \\
\hline \multicolumn{5}{|l|}{ Tatuagem } \\
\hline Sim & $2 / 12$ & 16,7 & 1.0 & \\
\hline Não & $21 / 48$ & 43,8 & $3,9(0,8-19,7)$ & 0,08 \\
\hline \multicolumn{5}{|l|}{ Uso de piercing } \\
\hline $\operatorname{Sim}$ & $14 / 36$ & 38,9 & 1.0 & \\
\hline Não & $9 / 24$ & 37,5 & $0,9(0,3-2,7)$ & 0,90 \\
\hline \multicolumn{5}{|l|}{ Uso de drogas lícitas } \\
\hline Sim & $5 / 19$ & 26,3 & 1.0 & \\
\hline Não & $18 / 41$ & 43,9 & $2,2(0,7-7,2)$ & 0,20 \\
\hline \multicolumn{5}{|l|}{ Uso de drogas ilícitas } \\
\hline $\operatorname{Sim}$ & $8 / 25$ & 32,0 & 1.0 & \\
\hline Não & $15 / 35$ & 42,9 & $1,4(0,7-2,6)$ & 0,50 \\
\hline \multicolumn{5}{|c|}{ Acesso a informações sobre sexualidade com os pais } \\
\hline Não & $11 / 38$ & 28,9 & 1.0 & \\
\hline Sim & $12 / 22$ & 54,5 & $1,9(1,0-3,5)$ & 0,05 \\
\hline \multicolumn{5}{|c|}{ Acesso a informações sobre sexualidade na escola } \\
\hline Não & $13 / 43$ & 30,2 & 1.0 & \\
\hline Sim & $10 / 17$ & 58,8 & $1,9(1,1-3,6)$ & 0,04 \\
\hline \multicolumn{5}{|c|}{ Acesso a informações sobre sexualidade na UBS ${ }^{\ddagger}$} \\
\hline Não & $6 / 20$ & 30,0 & 1.0 & \\
\hline Sim & $17 / 40$ & 42,5 & $1,4(0,7-3,0)$ & 0,30 \\
\hline
\end{tabular}

Rev. Eletr. Enf. [Internet]. 2017 [acesso em:______; 19:a03. Disponível em: http://dx.doi.org/10.5216/ree.v19.39041. 


\section{Acesso à Unidade Básica de Saúde (UBS)}

Apenas 38 participantes $(36,2 \%)$ relataram ter frequentado a UBS do assentamento nos últimos seis meses. Os motivos mais frequentes estão evidenciados na Tabela 2.

Tabela 2. Serviços utilizados na UBS pelos adolescentes e jovens escolares de um assentamento urbano de Goiânia, GO, Brasil, 2013.

\begin{tabular}{ccc}
\hline Serviço* & $\mathbf{N}$ & $\%$ \\
\hline Emergências & 26 & 24,8 \\
Consultas médicas & 18 & 17,1 \\
Vacinas & 11 & 10,5 \\
Consultas Odontológicas & 5 & 4,8 \\
Pegar preservativos & 2 & 1,9 \\
Pegar anticoncepcionais & 1 & 1,0 \\
Outros & 4 & 4,0 \\
\hline
\end{tabular}

* Variável de múltipla escolha (N=38); UBS: Unidade Básica de Saúde.

\section{DISCUSSÃO}

O cuidado à saúde na adolescência e juventude, fases do ciclo vital com características sexuais e reprodutivas análogas ${ }^{(6)}$, tem sido tema recorrente na produção de conhecimento, com vistas à busca de soluções que minimizem a vulnerabilidade desses indivíduos. Contudo ainda são escassos estudos em áreas de assentamento urbano formal ${ }^{(7-8)}$, especialmente em relação a comportamentos voltados para a sexualidade humana e uso de preservativo masculino.

Estudos evidenciam que indivíduos com sexarca precoce estão expostos a riscos relacionados à atividade sexual desprotegida, que pode ter como consequência o aumento do risco de infecção sexualmente transmissível, gravidez precoce, complicações obstétricas, ansiedade, processos familiares interrompidos, evasão escolar, entre outras ${ }^{(9-10)}$.

Dentre os adolescentes e jovens participantes desta investigação, 40,6\% já haviam iniciado vida sexual. De acordo com os dados apresentados na Pesquisa Nacional de Saúde Escolar (PeNSE) 28,7\% dos indivíduos referiram vida sexual ativa ${ }^{(10)}$. Ainda, estudo da Organização Mundial da Saúde (OMS) realizado na Europa e na América do Norte identificou que 26,0\% dos escolares jovens de até 15 anos já haviam iniciado relação sexual ${ }^{(11)}$.

Esses índices evidenciam que a iniciação sexual da população jovem tem acontecido precocemente, em particular quanto se trata de indivíduos de áreas de assentamento urbano, o que por sua vez alerta para a imaturidade, bem como dificuldade dessa população em adotar comportamentos sexuais seguros. Assim, ratifica a vulnerabilidade dos escolares assentados e a necessidade de estratégias preventivas de sensibilização e corresponsabilidade quanto ao uso regular do preservativo, minimizando assim, a aquisição e disseminação de $\operatorname{IST}^{(7)}$.

O método de barreira representa a principal estratégia de prevenção da gravidez e IST, sendo um método que, se bem utilizado, não prejudica a relação sexual, apresenta excelente custo-efetividade, é prático e amplamente discutido, incentivado e distribuído gratuitamente pelas mais diferentes unidades de saúde em todo o Brasil. Contudo, a não adesão ao uso regular do preservativo continua sendo um dos 
principais fatores de vulnerabilidade da população jovem mundialmente ${ }^{(11-12)}$.

Estudos nacionais apresentam divergências com os dados encontrados no assentamento urbano, em que somente $38,3 \%$ dos investigados reportaram utilizar preservativo em todas as relações. De acordo com o PeNSE (2012), 75,3\% dos adolescentes utilizaram preservativo ${ }^{(10)}$. Já um estudo conduzido em 20 países da Europa verificou uma tendência global do aumento do uso do preservativo na última relação, chegando a $80 \%{ }^{(13)}$. Concernente ao sexo biológico, nesse estudo, escolares do sexo masculino $(40,5 \%)$ sobressaíram ao afirmar fazer o uso do preservativo, contra $34,8 \%$ do sexo feminino, em concordância com outros estudos ${ }^{(14-}$ 16).

Segundo as Nações Unidas sobre HIV/AIDS (UNAIDS) ${ }^{(17)}$, as concepções sociais e culturais das relações de gênero muitas vezes podem restringir o acesso das adolescentes a informações e conhecimentos básicos, tornando-as passivas nas relações sexuais ou adotando comportamentos/atitudes que potencializam o risco dessas jovens na aquisição e disseminação de IST.

A assimetria de sexo e concepção de gênero nas relações entre os adolescentes é percebida na negociação, quando pessoas do sexo feminino não exige o uso do preservativo com receio de perder e/ou prejudicar os laços afetivos com sua parceira, provocando assim o término do relacionamento. Dessa forma, cabe aos atores sociais em hebiatria definir estratégias nas quais as relações de gênero são decisivas a partir do empoderamento das mulheres e sensibilização dos homens ${ }^{(16,18)}$.

A ausência de tatuagem se mostrou marginal $(p=0,08)$ como um fator associado à utilização do preservativo entre os indivíduos investigados. Embora sejam ainda incipientes estudos voltados à temática, essa prática pode denotar aos jovens a perspectiva de experimentação e libertação, o que por sua vez, pode indicar uma atitude de risco frente às situações de vivência da sexualidade ${ }^{(2,5)}$.

Quase a metade da população estudada reportou uso abusivo de drogas lícitas e ilícitas, colocando este grupo em situação de maior vulnerabilidade, exigindo assim intervenções voltadas para a prevenção e controle do álcool e outras drogas nos assentamentos.

Estudos têm demonstrado que indivíduos consumidores de álcool e outras drogas para fins de entretenimento, prazer ou como meio de "fuga da realidade", pode facilitar a exposição a comportamentos de riscos diversos, em especial em relações sexuais casuais e muitas vezes desprotegidas ${ }^{(5,19)}$, evidenciando a grande associação entre o padrão de comportamento de risco para a saúde sexual e reprodutiva na adolescência.

Nesta investigação, significativamente, o acesso às informações sobre sexualidade por meio dos pais tornou os adolescentes e jovens mais conscientes dos riscos e adeptos ao uso regular do preservativo (OR: 1,9; IC 95\%: 1,0-3,5; p=0,05). De fato, a comunicação é o pilar de sustentação familiar e um grande fator protetor, proporcionando uma ótima autopercepção do estado de saúde por parte da população jovem ${ }^{(11)}$. Todavia, é indispensável que os grupos familiares não somente informem sobre o uso do preservativo, mas também sobre o desenvolvimento e vivência de uma sexualidade segura, abordando as violências, gravidez indesejada, orientação sexual e as IST ${ }^{(20-21)}$. 
Da mesma maneira, o acesso a essas informações na escola se mostrou favorável para a conscientização dos indivíduos ao uso do preservativo masculino (58,8\%) (OR: 1,9; IC 95\%: 1,1-3,6 p= 0,04). Embora significativo esses dados poderiam ser ainda mais expressivos, se a instituição de ensino tivesse o Programa Saúde e Prevenção na Escola (SPE) implantado.

A PeNSE detectou que $89,1 \%$ dos escolares receberam informações sobre IST e Aids na escola, porém não é a realidade da população em estudo ${ }^{(16)}$. Uma investigação realizada no mesmo assentamento para identificar a percepção dos professores sobre a temática de educação sexual encontrou dificuldade destes em abordar esse assunto na escola devido à falta de conhecimento durante a formação, competência técnica e apoio dos familiares, sendo que somente $4,76 \%$ dos professores investigados realizavam alguma atividade juntamente com os pais ${ }^{(22)}$.

Cabe destacar que a escola tem sido apontada como importante espaço de intervenção sobre educação sexual, sendo o segundo lugar de maior acesso aos insumos, seguido dos serviços de saúde. Das escolas brasileiras, 63\% já trabalham com o tema IST e Aids e cerca de 50.000 participam do SPE ${ }^{(6)}$, auxiliando a comunicação com os adolescentes e jovens, pois desencadeia iniciativas de promoção da saúde, potencializando o acesso e a parceria das escolas com a Unidade de Saúde ${ }^{(9,22)}$. Entretanto quando a instituição de ensino está inserida em áreas de assentamentos, o índice de SPE decresce consideravelmente ${ }^{(22)}$, sendo que a instituição do assentamento em questão ainda não contempla o Programa Saúde na Escola (PSE) no seu Projeto Político Pedagógico, conforme estabelecido nos Parâmetros Curriculares Nacionais.

Com esses resultados do trabalho, vemos que o acesso à informação, tanto pelos pais, quanto pela escola é um indicador de proteção às IST. Assim, a temática sexualidade deve ser trabalhada, de forma transversal, nas instituições de ensino e contínua no ambiente familiar, sempre em parceria com os serviços de saúde ${ }^{(23)}$.

Nesse estudo, o acesso à informação na UBS não se mostrou significativo, contudo é um fator de proteção para o uso do preservativo. Somente 1,9\% dos investigados procuraram a unidade para apanhar preservativos, evidenciando a fragilidade do serviço e a relevante urgência de conscientização de mudança de atitude por parte da equipe de saúde do assentamento. Um estudo realizado com adolescentes identificou que $83 \%$ dos participantes nunca procuraram serviço de saúde para obter esclarecimentos sobre sexualidade, nem mesmo para a obtenção de preservativo(21).

Foi evidenciada que essa clientela procura assistência à saúde na UBS somente em situações de extrema necessidade, não se preocupando com a atenção primária, ou seja, a prevenção. Fato preocupante que requer investimentos, para que fatos que demonstram ineficiência do serviço de saúde local e conhecimento deficiente quanto ao uso correto do preservativo, como o rompimento do preservativo ter ocorrido em 54\% dos investigados não continue ocorrendo (dados não apresentados). Ratifica-se, então, que a não cobertura por programas de Atenção Básica à Saúde (ABS) em muitos assentamentos e a dependência de polos de concentração urbanos, tornam esses adolescentes e jovens entregues ao risco de 
comportamentos deletérios e aquisição de agravos à saúde ${ }^{(24)}$.

Considera-se indispensável que tanto os adolescentes e jovens quanto os envolvidos no seu acompanhamento, conheçam e pratiquem as legislações referentes aos direitos sexuais e reprodutivos e, sobretudo, que a educação em saúde seja um instrumento que ultrapasse os muros da escola, abarcando todos os equipamentos sociais existentes no assentamento. Convém salientar que esses direitos visam à garantia da vivência da sexualidade de forma mais orientada e livre de conceitos preestabelecidos pelos padrões morais, religiosos, sociais e culturais.

Por fim, acredita-se que o enfermeiro, como educador, possa contribuir holisticamente para o empoderamento dos adolescentes e jovens na implementação de medidas preventivas e, consequentemente, na efetivação de ações que possibilitem a redução da vulnerabilidade desses indivíduos às IST. Espera-se que este estudo contribua para a produção de conhecimentos que irão fornecer informações para o aperfeiçoamento técnico-científico dos profissionais, com vistas a discutir questões relativas à sexualidade e adesão ao preservativo dos indivíduos assentados.

Cabe destacar que o estudo possui algumas limitações, a despeito de todos os cuidados adotados, são possíveis vieses decorrentes da coleta de dados, uma vez que foi baseada no relato verbal, podendo a prevalência estar sub ou superestimada. Além disso, há as limitações inerentes aos estudos transversais, uma vez que não permitem o estabelecimento de relações de causa e efeito. A escassez de estudos que retratam o uso de preservativo em indivíduos de assentamento urbano formal pode ter comprometido a discussão, uma vez que os poucos estudos foram conduzidos em assentamentos informais e/ou rurais ${ }^{(8,25)}$.

\section{CONCLUSÕES}

Os adolescentes e jovens assentados se mostraram vulneráveis, com baixa adesão ao preservativo masculino, multiplicidade de comportamentos/atitudes de risco e baixíssima procura aos serviços de prevenção e promoção da saúde. Escolaridade acima de seis anos, acesso a informação sobre sexualidade com os pais e na escola se mostraram estatisticamente associado ao uso do preservativo, ratificando a relevância desses equipamentos sociais na manutenção da saúde desse grupo social.

Áreas de assentamento urbano formal necessitam de investimentos em políticas públicas que invistam no trabalho conjunto entre profissionais de saúde, familiares e instituições de ensino para atingirem com maior eficiência a conscientização desses indivíduos de sua vulnerabilidade às IST e demais questões relativas à sexualidade humana. Contudo, torna-se premente a realização de outros estudos em populações de assentamentos formais, tanto urbanos quanto rurais, uma vez que devido ao atual contexto social, político e econômico trata-se de um grupo emergente.

Por fim, os profissionais de saúde das UBS, em particular o enfermeiro, possuem papel importante no elo entre o adolescente, família e escola. Acredita-se que por meio da consulta de enfermagem em hebiatria no PSE, parece ser possível proporcionar um vínculo de confiança mútua para despertar na população jovem adesão a comportamentos de proteção e uma melhor qualidade de vida. 


\section{REFERÊNCIAS}

1. Patton GC, Sawyer SM, Santelli JS, Ross DA, Afifi R, Allen NB, et al. Our future: a Lancet commission on adolescent health and wellbeing. Lancet [Internet]. 2016 [acesso em: 15 abr. 2017];387(10036):2423-78. Disponível em: http://dx.doi.org/10.1016/s0140-6736(16)00579-1.

2. Morris JL, Rushwan H. Adolescent sexual and reproductive health: The global challenges. Int J Gynaecol Obstet [Internet]. 2015 [acesso em: 15 abr. 2017];131 Suppl 1:S40-2. Disponível em:

http://dx.doi.org/10.1016/j.jigo.2015.02.006.

3. Silva CR, Lopes RE. Adolescência e juventude: entre conceitos e políticas públicas. Cadernos de Terapia Ocupacional da UFSCar [Internet]. 2009 [acesso em: 15 abr. 2017];17(2):87-106. Disponível em:

http://www.cadernosdeterapiaocupacional.ufscar.br/index.php/cadernos/article/view/100.

4. Ayres JRCM. Organização das ações de atenção à saúde: modelos e práticas. Saude soc. [Internet]. 2009 [acesso em: 15 abr. 2017];18 Suppl 2:11-23. Disponível em: http://dx.doi.org/10.1590/S0104-12902009000600003.

5. Morales-Alemán MM, Scarinci IC. Correlates and predictors of sexual health among adolescent Latinas in the United States: A systematic review of the literature, 2004-2015. Prev Med [Internet]. 2016 [acesso em: 15 abr. 2017];87:183-

93. Disponível em: http://dx.doi.org/10.1016/j.ypmed.2016.03.005.

6. Ministério da Saúde. Recomendações para a Atenção Integral a Adolescentes e Jovens Vivendo com HIV/Aids [Internet]. Brasília: Ministério da Saúde; 2013 [acesso em: 15 abr. 2017]. Disponível em:

http://bvsms.saude.gov.br/bvs/publicacoes/recomendacoes_atencao_integral_hiv.pdf.

7. Kaufman ZA, Braunschweig EN, Feeney J, Dringus S, Weiss H, Delany-Moretlwe S, et al. Sexual risk behavior, alcohol use, and social media use among secondary school students in informal settlements in Cape Town and Port Elizabeth, South Africa. AIDS Behav [Internet]. 2014 [acesso em: 15 abr. 2017];18(9):1661-74. Disponível em:

http://dx.doi.org/10.1007/s10461-014-0816-x.

8. Kalolo A, Stephen MK. The influence of perceived behaviour control, attitude and empowerment on reported condom use and intention to use condoms among adolescents in rural Tanzania. Reprod Health [Internet]. 2015 [acesso em: 15 abr. 2017];12:105. Disponível em: http://dx.doi.org/10.1186/s12978-015-0097-5.

9. Santos TMB, Albuquerque LBB, Bandeira CDF, Colares VSA. Fatores que contribuem para o início da atividade sexual em adolescentes: revisão integrativa. Rev. de Atenção à Saúde [Internet]. 2015 [acesso em: 15 abr. 2017];13(44):64-

70. Disponível em: http://dx.doi.org/10.13037/ras.vol13n44.2668.

10. Souza LB, Martins P. Pesquisa Nacional de Saúde do Escolar 2015 [Internet]. 2015 [acesso em: 15 abr. 2017].

Disponível em: ftp://balcao.saude.ms.gov.br/horde/telessaude/apresentacao/2015/PeNSE2015.pdf.

11. Currie C, Zanotti C, Morgan A, Currie D, Looze M, Roberts C, et al. Social determinants of health and well-being among young people. Health Behaviour in School-aged Children (HBSC) study: international report from the 2009/2010 survey [Internet]. Copenhagen: WHO Regional Office for Europe, 2012 [acesso em: 15 abr. 2017]. Disponível em: http://www.euro.who.int/_data/assets/pdf_file/0003/163857/Social-determinants-of-health-andwell-being-among-young-people.pdf.

12. Costa AGM, Luna IT, Silva AA, Mesquita JS, Pinheiro PNC, Veira NFC. Percepção de saúde de adolescentes de comunidade rural: entre o ideal e o real. Rev. Eletr. Enf. [Internet]. 2013 [acesso em: 15 abr. 2017];15(4):870-7. Disponivel em: http://dx.doi.org/10.5216/ree.v15i4.19710.

13. Ramiro L, Windlin B, Reis M, Gabhainn SN, Jovic S, Matos MG, et al. Gendered trends in early and very early sex and condom use in 20 European countries from 2002 to 2010. Eur J Public Health [Internet]. 2015 [acesso em: 15 abr. 2017];25 Suppl 2:65-8. Disponível em: http://dx.doi.org/10.1093/eurpub/ckv030.

14. Malta DC, Silva MA, Mello FC, Monteiro RA, Porto DL, Sardinha LM, et al. Sexual health of adolescents according to the National Survey of School Health. Rev Bras Epidemiol [Internet]. 2011 [acesso em: 15 abr. 2017];14 Suppl 1:147-

56. Disponível em: http://dx.doi.org/10.1590/S1415-790X2011000500015.

15. Borges ALV, Fujimori E, Kuschnir MCC, Chofakian CBN, Moraes AJP, Azevedo GD, et al. ERICA: sexual initiation and contraception in Brazilian adolescents. Rev. Saúde Pública [Internet]. 2016 [acesso em: 15 abr. 2017];50 supl. 1:15s. Disponível em: http://dx.doi.org/10.1590/s01518-8787.2016050006686.

16. Oliveira-Campos M, Nunes NL, Madeira FC, Santos MG, Bregmann SR, Malta DC, et al. Sexual behavior among Brazilian adolescents, National Adolescent School-based Health Survey (PeNSE 2012). Rev Bras Epidemiol [Internet]. 2014 [acesso em: 15 abr. 2017];17 supl. 1:116-30. Disponível em: http://dx.doi.org/10.1590/18094503201400050010. 
17. UNAIDS Brasil [Internet]. UNFPA, OMS e UNAIDS: declaração de posição sobre preservativos e a prevenção do HIV, outras infecções sexualmente transmissíveis e gravidez indesejada. Brasília: UNAIDS Brasil, 2015 [acesso em: 15 abr. 2017]. Disponível em: http://unaids.org.br/2015/07/unfpa-oms-e-unaids-declaracao-de-posicao-sobre-preservativose-a-prevencao-do-hiv-outras-infeccoes-sexualmente-transmissiveis-e-gravidez-indesejada/.

18. Sampaio J, Santos RC, Callou JLL, Souza BBC. Ele não quer com camisinha e eu quero me prevenir: exposição de adolescentes do sexo feminino às DST/aids no semi-árido nordestino. Saude soc. [Internet]. 2011 [acesso em: 15 abr. 2017];20(1):171-81. Disponível em: http://dx.doi.org/10.1590/S0104-12902011000100019.

19. MacArthur GJ, Smith MC, Melotti R, Heron J, Macleod J, Hickman M, et al. Patterns of alcohol use and multiple risk behaviour by gender during early and late adolescence: the ALSPAC cohort. J Public Health (Oxf) [Internet]. 2012 [acesso em: 15 abr. 2017];34 supl. 1:i20-30. Disponível em: http://dx.doi.org/10.1093/pubmed/fds006.

20. Santa Maria D, Markham C, Bluethmann S, Mullen PD. Parent-based adolescent sexual health interventions and effect on communication outcomes: a systematic review and meta-analyses. Perspect Sex Reprod Health [Internet]. 2015 [acesso em: 15 abr. 2017];47(1):37-50. Disponível em: http://dx.doi.org/10.1363/47e2415.

21. Brum MM, Carrar K. História individual e práticas culturais: efeitos no uso de preservativos por adolescentes. Estud. psicol. (Campinas) [Internet]. 2012 [acesso em: 15 abr. 2017];29 supl. 1:689-97. Disponível em:

http://dx.doi.org/10.1590/S0103-166X2012000500005.

22. Carvalho PMRS, Guimarães RA, Moraes PÁ, Teles SA, Matos MA. Prevalence of signs and symptoms and knowledge about sexually transmitted diseases. Acta paul. enferm. [Internet].2015 [acesso em: 15 abr. 2017];28(1):95-100. Disponível em: http://dx.doi.org/10.1590/1982-0194201500016.

23. Chaveiro LG, Pires LM, Matos MA, Teles SA, Souza SMB, Souza MM. Thematic analysis of sexuality in the school context with teachers of basic education. Rev Rene [Internet]. 2015 [acesso em: 15 abr. 2017];16(5):690-8. Disponível em: http://dx.doi.org/10.15253/2175-6783.2015000500010.

24. Cortez R, Saadat S, Marinda E, Odutolu O. Adolescent fertility and sexual health in Nigeria. Health, Nutrition and Population (HNP) Discussion Paper [Internet]. Washington, D.C.: World Bank Group, 2016 [acesso em: 15 abr. 2017]. Disponível em: http://documents.worldbank.org/curated/en/507641468190770251/Adolescent-fertility-and-sexualhealth-in-Nigeria.

25. Muhula S, Memiah P, Mbau L, Oruko H, Baker B, Ikiara G, et al. Uptake and linkage into care over one year of providing HIV testing and counselling through community and health facility testing modalities in urban informal settlement of Kibera, Nairobi Kenya. BMC Public Health [Internet]. 2016 [acesso em: 15 abr. 2017];16:373. Disponível em: http://dx.doi.org/10.1186/s12889-016-3033-x. 